Sección uno: Ensayo

Retos y Experiencias en la Construcción de Paz

\title{
Conflictos de la diversidad cultural y la cultura de paz en Latinoamérica ${ }^{1}$
}

Conflicts of cultural diversity and culture of peace in Latin America

\author{
Eulogio García Vallinas \\ Universidad de Cádiz (España) \\ eulogio.garcia@uca.es \\ Maury Almanza Iglesias \\ Universidad Simón Bolívar \\ (Colombia) \\ malmanza@unisimonbolivar.edu.co
}

\section{Resumen}

Se describen los conflictos de la diversidad cultural en Latinoamérica desde la compresión tanto de las dinámicas y consecuencias étnico-culturales producidas por los procesos históricos de colonización, independencia y conformación de los Estados Nacionales; como, en la actualidad, de la renovada complejidad y conflictividad que el proceso de globalización y las políticas neoliberales introducen en dichas dinámicas para las personas, grupos étnicoculturales, colectivos sociales y comunidades, desde los referentes conceptuales y el horizonte valórico de la cultura de paz.

\begin{abstract}
The conflicts of cultural diversity in Latin America are described from the understanding of both the dynamics and ethnic-cultural consequences produced by the historical processes of colonization, independence and conformation of the national States; as, at present, the renewed complexity and unrest that the process of globalization and neoliberal policies introduce in these dynamics for people, ethnic-cultural groups, social groups and communities, from the conceptual referents and the values horizon of the culture of peace.
\end{abstract}

${ }^{1}$ Recibido: 19/09/2018 Evaluado: 25/09/20118 Aceptado: 10/10/2018 
Palabras clave: Diversidad cultural, conflictos, cultura de paz, Latinoamérica.

Key words: Cultural diversity, conflicts, culture of peace, Latin America.

\section{Introducción}

Cabría afirmar que la diversidad cultural ha sido una constante desde la conformación de las primeras sociedades humanas y rasgo esencial de estas (Gellner, 1997). Es elemento constitutivo de la identidad de América Latina desde sus orígenes. Diversidad que fue haciéndose más compleja y rica a lo largo de la historia por los importantes flujos migratorios y la proliferación identitaria que se fue gestando y amalgamando en esos procesos, a pesar de los esfuerzos de las instituciones por negarla e invisibilizarla, de manera especial la relativa a las poblaciones indígenas y afrodescendientes, considerándola históricamente un obstáculo a la constitución de Estados modernos, que fueron configurándose como uninacionales sobre unidades multiétnicas y pluriculturales. Sin embargo hoy los valores de diversidad, pluralismo, interculturalidad... orientan políticas públicas y ocupan un lugar central en las preocupaciones y recomendaciones de organismos internacionales, en foros e instrumentos regionales y en las reformas legislativas más recientes de todos los países latinoamericanos en temas de cultura, desarrollo social, justicia, educación, salud y medio ambiente (UNESCO, 1996, 2001, 2005; PENUD, 2004). Pero a pesar del amplio consenso social generado sobre la importancia de estos temas siempre existirá margen para ampliar la comprensión de los mismos y abrir espacios en los que se pueda debatir el mejor modo de gestionarlos. Aquí reflexionaremos sobre ellos desde referentes de la Cultura de Paz y de la investigación sobre la paz y los conflictos, intentando dilucidar posibilidades de empoderamiento y reconocimiento a través de la transformación pacífica de conflictos.

\section{Diversidad cultural y geoestrategia}

Desde posiciones teóricas diferentes se percibe que tanto el refuerzo de las identidades como las controversias culturales tendrán un papel relevante en el escenario social de las próximas décadas. Huntington (1996) advirtió sobre los cambios en las identidades de los pueblos y la reconfiguración de la política global en torno a lineamientos culturales después de la guerra fría, ubicando la línea de fractura de los conflictos entre las etnicidades construidas o reinventadas y entre civilizaciones. Por su parte Wallerstein (1996) señala al factor cultural como una dimensión importante en la crisis del sistema-mundo, debido al creciente cuestionamiento de la ideología universalista que acompañó al capitalismo. Afirma que la alternativa a la crisis sistémica no vendrá de la mano del individuo, sostenido por el liberalismo, sino del fortalecimiento de los grupos de identidad. Cuando menos estas tesis pueden ser contempladas como síntomas de las incertidumbres suscitadas por el renacimiento de las identidades, los movimientos en favor de políticas de reconocimiento de la multiculturalidad y los enfrentamientos étnico-nacionales en todo el mundo. 
Como rasgo esencial de la sociedad humana la diversidad plantea algunos problemas, como el de decidir cuál de las formas culturales que coexisten en un determinado momento y lugar es mejor, a qué responden las diferencias y cómo afrontar las consecuencias de la misma. Estas cuestiones están presentes desde que los grupos sociales se fueron haciendo más complejos, con la inclusión en su seno de varias configuraciones culturales, compartiendo el sistema político y económico, en organizaciones jerárquicas. La diversidad empieza a ser un factor potencial de conflictos y problemas intrasocietales, surge la otredad sociocultural como problema. Por un lado tendríamos lo que un sujeto tiene que hacer como miembro de una cultura y, por otro, lo que tiene que asumir, por estar en el contexto de otra que es receptora o dominante.

A lo largo de la historia se ponen de manifiesto en esos contextos complejos los esfuerzos constantes de la cultura dominante por manejar, controlar o suprimir la diversidad cultural. Hemos podido constatar la dificultad, si no imposibilidad, de suprimir la diversidad cultural, pues se manifiesta como un rasgo sustantivo de la sociedad y consustancial al ser humano, lo que hace muy difícil anularla.

\section{Discursos negacionistas, procesos históricos y políticas de Estado excluyentes.}

Existen discursos que niegan o minimizan la diversidad étnica y cultural de América Latina como los que la consideran una apelación nostálgica al pasado, un intento romántico y trasnochado de recuperar tradiciones exóticas que alimentan los folklores locales. Otros se basan en un evolucionismo acrítico que percibe el presente como el culmen de la experiencia civilizatoria humana. El más extendido quizá sea el de quienes consideran que el humanismo genérico pasa por la abolición de las diferencias, cuyos rasgos coinciden con los de la civilización occidental. Una corriente a la que se adscriben de modo convergente políticas estatales y las homogeneizadoras dinámicas del mercado, que demandan consumidores con apetencias similares; al igual que ciertos sectores radicales que identifican homogeneidad con igualdad.

Las poblaciones indígenas y afrodescendientes en América Latina presentan historias y contextos regionales diversos que han condicionado su devenir histórico hasta hoy. Han sido víctimas de la construcción de estados nacionales modernos a imagen del modelo teórico de la Ilustración, para el que un Estado debía corresponder con una comunidad culturalmente homogénea. Este argumento alentó las políticas indigenistas que perseguían la integración de esas poblaciones en la comunidad nacional. La diferencia constituía una afrenta al modelo estatal, por lo que se promovió la asimilación a ese modelo imaginario. La represión cultural y lingüística, la aniquilación cultural o el etnocidio deliberado constituyeron objetivos de las políticas públicas de unos Estados que actuaron durante los siglos diecinueve y veinte como formaciones neocoloniales.

Las independencias fueron procesos liderados por criollos que a pesar de utilizar en las contiendas militares a poblaciones indígenas y afrodescendientes no compartieron con ellas el protagonismo de la configuración nacional de las nuevas repúblicas, cuyo diseño respondió al modelo europeo seguido por la minoría blanca o mestiza que se hizo con el poder e inició una expansión neocolonial sobre tierras indígenas, consolidando su posición como clase privilegiada de grandes terratenientes. Otro momento de intensa ocupación de tierras 
indígenas se dio con el desarrollismo de la segunda mitad del siglo veinte por las posibilidades de explotación de bosques y minerales. Las políticas neoliberales de los Estados en la actualidad excluyen a las poblaciones indígenas de su modelo económico, que no los requiere siquiera como fuerza de trabajo.

\section{Pobreza y diversidad cultural: la otredad ausente}

Como resultado de esos procesos históricos a los que aludíamos los pueblos indígenas ocupan las posiciones más bajas en las escalas de indicadores sociales y económicos en Latinoamérica. En torno al $80 \%$ de los indígenas vive bajo la línea de la pobreza. Stavenhagen (2003) sostiene que lejos de ser un hecho casual la pobreza y la extrema pobreza de las poblaciones indígenas responde al efecto acumulado de políticas discriminatorias, las cuales dejan al descubierto la perspectiva racista que ha guiado las acciones de los grupos dominantes desde la época colonial, agudizadas si cabe por el darwinismo social y cultural de las políticas neocolonialistas de los siglos diecinueve y veinte.

Las posibilidades transformadoras de la precariedad en la actualidad deberían pasar por la redefinición de las relaciones interétnicas tanto en el campo cultural como en el político y económico. Debe cambiar la lógica estatal que se dirige a una ciudadanía arbitrariamente uniformada para dar cabida a la presencia de sujetos colectivos y, como tales, portadores de derechos colectivos; debe garantizar el reconocimiento del ejercicio de la diferencia por parte de colectividades culturalmente diferenciadas. Ello no pasaría sólo por una tolerancia filosófica (Taylor, 1993 y 1994), sino por el ejercicio pleno de sus sistemas políticos, económicos, sociales, cosmológicos y culturales, incluyendo el derecho a una autonomía territorial que otorgue un marco espacial a la reproducción de la colectividad (Bartolomé, 2008, p. 40). La pobreza de muchas comunidades étnicas en América Latina tiene que ver con la apropiación que el Estado o los concesionarios empresariales han hecho de sus territorios remanentes, imposibilitando su acceso a recursos del suelo y del subsuelo: ríos, selvas, bosques, acuíferos, plantas medicinales, el uso de semillas autóctonas, etc.

Según Bartolomé (1988), durante las generaciones coloniales y neocoloniales las poblaciones indígenas desarrollaron una cultura de la resistencia que permitió su reproducción y supervivencia en condiciones en las que parecía casi imposible; su censo hoy está en torno a 45 millones de personas, y el de afrodescendientes se aproxima a 150 millones. No se puede considerar a estas culturas como sobrevivientes del pasado, pues han operado procesos históricos de transfiguración étnica y cultural en los que han reconfigurado las culturas existentes en el momento del contacto; es decir, las culturas cambian, evolucionan, se proyectan hacia el presente y al futuro. Según este autor, los últimos registros etnográficos reflejan también procesos de re-etnificación de poblaciones que habían dejado de ser consideradas y de considerarse así mismas en términos étnicos por no conservar lengua propia, pero siendo este un referente fundamental de la identidad también se puede apelar a otros importantes como la historia, el territorio, la comunidad, las tradiciones compartidas, las prácticas rituales, organizacionales, etc. que las identifican también como comunidades identitarias. En otros casos la re-etnificación proviene de una nueva dignidad que emerge de su participación activa en procesos políticos nacionales, como está ocurriendo en México, Bolivia, Ecuador; procesos que influyen positivamente en la redefinición de la 
autopercepción colectiva devaluada anterior, en los cuales sujetos y comunidades se empoderan.

Estas dinámicas de resistencia cultural y re-etnificación ponen de manifiesto cómo la diversidad en América Latina se multiplica y amplía, lejos de reducirse y ajustarse a los estrechos parámetros impuestos por los Estados. En este sentido apuntan también los datos migratorios intra y extra-regionales, pues las comunidades migrantes pasan a ser percibidas y a percibirse en las sociedades receptoras como grupos organizacionales diferenciados, generando sus propias fronteras interactivas de acuerdo a los diferentes contextos de convivencia, contextos en los que la diversidad es la norma y no un fenómeno coyuntural.

\section{Identidades múltiples}

Estas realidades aportan argumentos para superar las tentaciones etnocéntricas y aislacionistas que acechan a la identidad y para asumir una noción de la misma que rechace todo supuesto esencialista sobre las culturas. Lo que implica entenderlas antes que nada como históricas, pues se conforman en contextos complejos en los que están presentes otras culturas respecto de las cuales se define la propia pertenencia. Esos contextos son también cambiantes y hacen que las identidades se transformen, pues no son inmunes a los desafíos procesuales de todo tipo, evolucionan. Las identidades, por lo tanto, son dinámicas, cambian, se adaptan y experimentan todo tipo de ajustes internos, se moldean en contacto con otras culturas. Las identidades son internamente heterogéneas, no están exentas de tensiones y conflictos a resolver. En su seno hay subgrupos, se plantean distintas opciones, tienen contradicciones y problemas. Una heterogeneidad que posibilita la autorreflexion, la crítica, el disenso y no solo el consenso o la adhesión (Díaz-Polanco, 2000).

Este autor las define como identidades múltiples. Identidades de grupos incluidos en sociedades mayores como el Estado-nación, donde la identidad se vive intersubjetivamente. Las identidades múltiples también pueden consolidarse o expandirse, presionar las fronteras establecidas, redefiniéndolas. Estos procesos son perceptibles en la fase actual de reestructuración de los Estados nacionales, en la que las fronteras en el ámbito regional se hacen más porosas. Se puede afirmar que las identidades múltiples son la regla, pues los sujetos no nos adscribimos a una identidad única, sino a una multiplicidad de pertenencias que nosotros mismos organizamos y jerarquizamos dentro de las posibilidades o en el marco de restricciones sistémicas, pero que están presentes en nosotros de modo simultáneo. Por ello, para comprender la diversidad es necesario atender a la articulación compleja de los diferentes planos identitarios que constituyen nuestra dimensión social. Todas estas capas y sus influencias mutuas dan sentido a la adscripción cultural. Sin embargo, esta multiplicidad de capas o planos opera bajo un principio de jerarquía. Su importancia subjetiva o intersubjetiva puede variar en cada caso o momento. Aunque tampoco la jerarquización identitaria es estática. Jerarquías distintas pueden operar en circunstancias diferentes: en un momento puede primar la etnia, en otro el género, en otro la filiación religiosa o la nacional, etc. La noción de identidad múltiple, matizada por el principio de jerarquización identitaria, permite comprender que una determinada adscripción identitaria no supone necesariamente rechazo de otras pertenencias con las que puedan compartirse horizontes.

\section{Comunidad vs globalización}


Díaz-Polanco (2000 y 2009) alerta sobre la pretensión de utilizar la noción de identidades múltiples para desvalorizar la identidad misma, contemplándola bajo la perspectiva de la "fluidez" o el "hibridismo" que relativizan el sentido de pertenencia pues, aunque también exista este tipo de identidades múltiples que la globalización capitalista trata de generalizar, no son las únicas posibles. Bauman (2005 y 2013) las denomina comunidades de guardarropa, que denota los rasgos de estas uniones temporales, como fenómenos de identificación de recorrido breve, como eventos puntuales o espectáculos a los que los individuos asisten y al concluir la función se disuelven. El autor caracteriza este periodo de cultura globalizada como modernidad líquida, en la que los espectáculos sustituyen a la causa común de la modernidad sólida, ofreciendo a los individuos identidades volátiles materializadas en experiencias colectivas concertadas y armoniosas, aliviando la soledad que esas comunidades reproducen en sustitución de las comunidades genuinas, que son duraderas y abarcadoras. De este modo, lejos de ponderar la complejidad, profundidad y sentido de la identidad, sólo tratan de limar sus aristas y restar importancia al fenómeno identitario. Identidad y comunidad son fenómenos entrelazados. La identidad básica se asocia a la comunidad, pues el sentido de pertenencia fluye de la comunidad y cuando el sentido de comunidad desaparece las identidades múltiples entran en crisis, se transforman en identidades agónicas, con referentes volátiles fácilmente manipulables por el mercado o por la política.

La otra comunidad es la que aporta a los sujetos sentido duradero y profundo, se funda en tejidos y nexos sociales con alguna referencia espacial, territorial, situada, y en cuyo ámbito puedan construir tanto identidades sólidas como proyectos compartidos de alcance social. Este es el tipo de comunidad que puede resistirse a los efectos del poder globalizado. El papel de la resistencia política en el ámbito cultural supone la defensa de una forma de vida propia, identificando las formas visibles e invisibles de imposición cultural, visualizando riesgos y fuerzas destructivas (Jameson, 2000) o, como sugiere Fornet-Betancourt (2003), "desculturizando la cultura" que generan grupos sociales dominantes para sacralizar ciertas tradiciones como las "propias" y excluir otras como "inauténticas", explicitando la asimetría que refleja el nombrar unilateralmente "lo propio" y la exculturación de posibilidades que pudieron igualmente ser propias. Sin que ello signifique un reclamo conservador a la tradición, nuevas intolerancias o autoritarismos. Las políticas emancipatorias deben evitar caer en fundamentalismos en defensa de la comunidad democrática, abierta, innovadora, incluyente y con autonomía. Es en la construcción de comunidad donde podemos encontrar las claves para encarar las amenazas globalizadoras, así como pensar y realizar tentativas de otro mundo posible.

\section{Los conflictos de la diversidad desde la Cultura de Paz}

Pensar en términos de paz positiva significa descartar todas las formas de violencia en las estructuras sociales y la realización de la justicia social. En otras palabras, significa un estado de liberación. Siguiendo a Galtung (2003) la paz es ausencia de violencia, injusticia, inequidad y opresión; además de un estado en el que las necesidades humanas fundamentales estén siendo satisfechas, así como crear activamente las condiciones de paz para prevenir la violencia. ¿Cómo buscar formas pacíficas de evitar el sufrimiento que unos seres humanos nos causamos a otros? Como plantea Galtung, este sufrimiento puede ser personal, estructural o basado en la violencia cultural o simbólica, y tiene también una dimensión 
ecológica. Hacer sufrir es hacer que unos seres humanos lleven su vida por debajo de sus capacidades, potencialidades, o competencias como seres humanos; que pierdan humanidad, se degeneren.

La interacción e interdependencia es una muestra de la fragilidad, por la que a menudo desconocemos las consecuencias de nuestras acciones (Arendt,1996). El descubrimiento de la interdependencia humana puede producir miedo (Reardon, 1985) a las diferencias entre personas, grupos de identidad, culturas y a la alteridad de la misma tierra. A menudo este miedo está entre las causas profundas de los conflictos. La cara positiva del mismo expresa la fragilidad y vulnerabilidad humana y la necesidad de la otredad, de interrelación e interdependencia.

\section{La transformación positiva de los conflictos de la diversidad}

Las políticas para afrontar ese miedo pueden basarse en el ejercicio de la dominación, la exclusión, marginación y hasta anulación de cualquier tipo de diversidad con el ejercicio de la violencia; en cuyo caso diríamos que no hemos sido capaces de gestionar pacíficamente los conflictos. Concebir la competencia y capacidad humana para construir paz es reconocer la propia identidad personal y colectiva para actuar desde la interacción con otras identidades y grupos. Supone renunciar a considerar al ser humano o los pueblos como identidades absolutas centradas en sí mismas. Lo mismo que podemos producir interdependencia para crear esas identidades podemos superar los procedimientos violentos para ser parte de la condición humana. Podemos organizarnos políticamente asumiendo nuestra fragilidad para involucrarnos por medios pacíficos en la transformación de los conflictos que la interdependencia pueda generar (Martínez Guzmán, 2005).

Una cuestión crítica relacionada con la interdependencia y la interacción es la de la asimetría, que a menudo las cuestiona. Por ejemplo, la gente tiene derecho a comer y no morir de hambre, pero también a hacerlo desde sus propios valores, saberes, culturas y creencias; desde el derecho a su propia identidad en interacción, y no dominados por otras identidades; Sen (2000) lo denomina desarrollo con libertad o derecho a desarrollarse desde el ejercicio libre de sus capacidades y desde la consideración de sus propios valores. También se plantea la necesidad de deconstruir lo que tengan de marginación y exclusión de unos seres humanos por otros, de unas por otras identidades, culturas, comunidades, poblaciones; o lo que tengan de depredación de la naturaleza; para reconstruir las capacidades de interacción humana por medios pacíficos.

\section{Empoderamiento y reconocimiento}

Como venimos argumentando, los seres humanos, individualmente y en grupo, tenemos poderes o capacidades para organizarnos, excluirnos, destruirnos y hacernos la guerra; pero también tenemos poderes y capacidades para hacer políticas que conviertan el miedo a la alteridad en asunción de la fragilidad y la vulnerabilidad como expresión de la interdependencia. Una concepción del ser humano basada en su capacidad para performar o configurar las relaciones en un sentido u otro. El empoderamiento consistiría en realizar políticas en las que los propios seres humanos y los colectivos puedan ejercer sus poderes, 
capacidades y competencias desde sus culturas, saberes, valores y creencias, en interacción con las de los otros y las otras y formando parte de la tierra (Martínez Guzmán, 2006).

En relación con el reconocimiento emergen al menos dos maneras de abordarlo. Una ligada al reconocimiento de los derechos colectivos en el marco del debate del multiculturalismo (Taylor, 1994); y otra que promueve el reconocimiento basado en el amor, el respeto de los derechos, la estima y la solidaridad de la diversidad de las formas de vida (Honneth, 1997). Ambas coinciden en la necesidad de promover "luchas por el reconocimiento" por medios pacíficos como alternativas a las luchas enredadas en la espiral de la violencia, como las de los conflictos armados y estructurales. Sin detenernos en el debate de los derechos colectivos a la propia lengua, creencias, cultura o cualquiera de las características de la identidad colectiva, conviene precisar la distinción entre multiculturalidad y multiculturalismo para referirnos a la diversidad cultural. La multiculturalidad expresa el reconocimiento de la existencia de múltiples culturas, mientras que el multiculturalismo sería el sostén teórico de ese reconocimiento, que puede llevar a un relativismo en el que "todo vale" sólo por ser diferente, llegándose a proponer la "inconmensurabilidad" de las culturas.

Desde la perspectiva de la performatividad de la condición humana, que nos capacita para configurar las relaciones de múltiples formas, siempre podremos pedirnos cuentas por lo que hacemos, decimos o callamos, y ello supondría dar sentido y profundizar en el concepto de "interculturalidad", enraizado en la pluralidad de la condición política del ser humano. La vida política demanda que seamos suficientemente iguales para poder entendernos y lo suficientemente diferentes para activar nuestra capacidad de comunicación, de otro modo no tendríamos nada que decirnos, no habría discurso. La interculturalidad como instrumento del reconocimiento para la transformación pacífica de los conflictos consiste en la interpelación mutua, desde nuestra igualdad y diferencia, como ejercicio de nuestras capacidades o poderes de comunicación.

El reconocimiento como interculturalidad, como interpelación y no choque, en el sentido de Huntington (1997), de culturas, civilizaciones, identidades colectivas y personales, se basa precisamente en aquella asunción de la fragilidad y vulnerabilidad que muestra la necesidad de interrelacionarnos e interaccionar. Hay una relación necesaria entre autoconciencia y reconocimiento intersubjetivo (Honneth, 1997). Se basa en la asunción de que la configuración de la propia identidad personal y colectiva sólo puede darse en el marco de esa interrelación e interacción, en la que no "todo vale" por ser diferentes; al contrario, todo estaría sometido a la interpelación mutua, desde el reconocimiento recíproco, como interlocutores con iguales derechos a ejercer sus capacidades de comunicación.

En el marco de las luchas por el reconocimiento se alerta sobre la posibilidad de que no exista simetría en la interlocución, debido a desigualdades sociales, marginación o exclusión (Fornet-Betancourt, 2003; Dusell, 2003). Entonces habría que activar formas de empoderamiento para que todos tengan la oportunidad de ejercer sus capacidades y competencias. En este sentido, importa menos la existencia de "una comunidad ideal de comunicación", atendiendo a la Ética del Discurso, que la atención al sufrimiento real de marginados y excluidos; lo que interpela a quienes pudiéramos tener la responsabilidad moral de facilitar las oportunidades para su propio desarrollo y ejercicio de sus competencias. 
Se trata también de reconocer la pluralidad de valores, formas de vida, culturas y creencias, denunciando aquella dominación de un único saber (occidental, blanco, masculino, del norte) que convierte a los demás saberes en "saberes sometidos", excluidos y marginados (Honneth, 1997; Lander, 2003). Como "no todo vale", tan erróneo sería la imposición de unos determinados valores por "considerarlos más desarrollados", como la aceptación de cualquier propuesta cultural o creencia simplemente porque "es diferente". En este caso el desprecio de las formas de vida diferentes produce o la reacción "fanática", o la falta de estima por los propios valores, culturas y creencias; como ocurría en grupos y comunidades indígenas y afrodescendientes en los procesos de re-etnificación cuando recuperaban su capacidad de agencia.

Otro ámbito conflictivo para Honneth tiene que ver con el reconocimiento de derechos jurídicos, en tanto que diferencia a quienes acceden y pueden ejercer esos derechos de los que no. Se refiere a la necesidad de que se nos reconozca como sujetos moralmente responsables de nuestros actos. En ese caso, las personas o grupos que dejamos fuera o en los márgenes "se crean su propia legalidad", separada de la legalidad oficial. Es una forma de excluir, de lo que consideramos nuestra comunidad moral, a aquellas personas o grupos a quienes no reconocemos los derechos morales y jurídicos que nosotros nos atribuimos.

La preocupación por el sufrimiento puede expresarse también en términos de liberación o emancipación de todo aquello que "somete" a otros seres humanos y a la propia naturaleza. Desde este compromiso crítico con la emancipación se ha producido un interesante debate entre Nancy Fraser y Axel Honneth (2006). Hemos pretendido hacer compatible la teoría del reconocimiento en Honneth $y$ en los estudios potscoloniales y subalternos latinoamericanos con la preocupación por la asimetría que se da en los procesos de reconocimiento y que requieren nuevas formas de justicia, o como dice el título del debate entre ambos autores, de "redistribución". En relación con el reconocimiento, Fraser trata de superar la falsa antítesis que parece producirse en la bibliografía internacional entre las "políticas del reconocimiento", la identidad y las culturas, y las "políticas basadas en la noción de clase social" más ligadas a la redistribución de recursos. Parece que la justicia social habría de decantarse por una u otra. Sin embargo la autora construye un modelo de justicia social en el que ambos elementos son fundamentales: hay que combinar el análisis de la desigual distribución sistémica de recursos enraizada en las estructuras económicas, con la falta de reconocimiento que tiene sus raíces en la subordinación de unos grupos por otros. La justicia social requiere ambas dimensiones: redistribución y reconocimiento.

\section{Conclusiones}

En línea con los estudios y análisis citados, referidos tanto al ámbito latinoamericano concreto como al más general de la comunidad internacional, la integración de la diversidad cultural en la amplia variedad de las políticas públicas parece hoy más urgente y necesaria que nunca antes por entenderse directamente vinculada al desarrollo sostenible de los pueblos, a la consolidación de la paz y a la prevención o superación pacífica de los conflictos.

La cultura es entendida como una dimensión transversal vinculada a los tres pilares de la sostenibilidad: economía, entorno social y ecología; los cuales han de articularse a través de políticas y prácticas situadas que atiendan a la especificidad del contexto. El enfoque de la 
diversidad está contribuyendo a la adopción de estrategias novedosas de gestión y preservación de los recursos naturales, como ocurre con los aportes y conocimientos de las comunidades indígenas, que nos muestran modos de vida más sostenible. Este enfoque desvela también que la pobreza es una violación intolerable de los derechos humanos que impide a las personas, grupos y comunidades afectados el desarrollo de una vida digna. De modo que la políticas públicas deberían atender a las circunstancias sociales y culturales de cada caso.

Parece necesario afrontar la reducción del analfabetismo cultural en un mundo globalizado donde los contactos entre las culturas se multiplican con rapidez. La capacidad para tolerar las diferencias culturales y de aceptarlas, en lugar de dejarse desestabilizar por ellas, demanda competencias interculturales que deberían figurar entre los propósito prioritario de la educación para la ciudadanía en cualquier contexto y entre las preocupaciones urgentes de las políticas públicas en general.

En relación con la paz y la prevención o superación pacífica de los conflictos, el reconocimiento de la diversidad cultural implica poner el énfasis en lo que nos une como personas, en la unidad dentro de la diversidad, en la humanidad compartida que es intrínseca a las diferencias humanas. Siendo la diversidad cultural fundamental para los derechos humanos debería producirse la apropiación de los mismos a nivel local, no como algo impuesto sobre las prácticas culturales sino como principios consustanciales a dichas prácticas y reflejo de nuestra humanidad compartida.

Parece conveniente explorar el nuevo enfoque que se abre con el reconocimiento de las identidades múltiples y multidimensionales de las personas y grupos para reforzar el desarrollo del pluralismo cultural, pues a la gente le cuesta cada día más aceptar ser clasificada en función de categorías preestablecidas, ya sean estas de componente étnico, lingüístico, cultural, político o de otro tipo; resistiéndose a ser clasificadas como identidades unidimensionales. Este hecho no cuestiona el significado de las identidades colectivas, pero sí da cuenta de la creciente movilidad e intercambio entre colectivos y grupos culturales que caracteriza las experiencias individuales de las personas, lo que contribuye poderosamente a la plasticidad de sus identidades $\mathrm{y}$, por ende, al diálogo intercultural.

El reconocimiento de la diversidad cultural afianza el ejercicio pleno de los derechos humanos, fortalece la cohesión social e inspira nuevas formas de gobernanza democrática. Todos los derechos y libertades se ejercen en contextos culturales muy diversos, tienen una dimensión cultural que es necesario reconocer para su integración efectiva en los diversos contextos culturales. Hoy resulta imposible negar la composición multicultural de las sociedades sin que ello suponga dañar el tejido social, excluir a amplios sectores de la población, segmentándola y obligando a las diferentes comunidades o colectivos a competir por el acceso a recursos como la educación, la sanidad, los servicios sociales, etc.; en lugar de hacerlos comunes y fomentar el valor de la solidaridad. Por otro lado, las formas de gobernanza democrática pueden renovarse con las aportaciones procedentes de los modelos adoptados por las distintas culturas. 


\section{Referencias}

Arendt, H. (1996). La condición humana. Barcelona: Ediciones Paidós.

Bartolomé, M.A. (2008). La diversidad de diversidades. Reflexiones sobre el pluralismo cultural en América Latina. Cuadernos de Antropología social, no 28, 33-49.

Bauman, S. (2005). Identidad. Madrid: Losada.

Bauman, S. (2013). La cultura en el mundo de la modernidad líquida. Buenos Aires: Fondo de Cultura Económica.

Díaz-Polanco, H. (2000). Los dilemas de la diversidad. Diálogos Latinoamericanos, nº 2 , 77-91.

Díaz-Polanco, H. (2003). Diez tesis sobre identidad, diversidad y globalización. Disponible en http://cort.as/-BPDk

Dussel, E. (2003). La globalización y las víctimas de la exclusión desde la perspectiva de la ética de la liberación. En R. Fornet-Betancourt: Culturas y poder. Interacción y asimetría entre las culturas en el contexto de la globalización. Bilbao: Desclée, pp.109-138.

Fornet-Beancourt, R. (2003). Interacción y asimetría en las culturas en el contexto de la globalización. En: Culturas y poder. Interacción y asimetría entre las culturas en el contexto de la globalización. Bilbao: Desclée, pp. 15-27.

Fraser. N y Honneth, A. (2006). ¿Redistribución o reconocimiento? Madrid: Morata.

Galtung, J. (2003). Paz por medios pacíficos. Bibao: Bakea, Centro de Documentación de Estudios para la Paz.

Gellner, E. (1997). Antropología y política. Revoluciones en el bosque sagrado. Barcelona: Gedisa.

Honneth, A. (1997). La lucha por el reconocimiento. Por una gramática moral de los conflictos sociales. Barcelona: Crítica.

Huntington, S.P. (1996). El choque de las civilizaciones. La reconfiguración del orden mundial, Barcelona: Paidós.

Jameson, F. (2000). Globalización y estrategia política. New Left Review, 5. Madrid: Akal.

Lander, E. (Comp.) (2003). La colonialidad del saber: eurocentrismo y ciencias sociales. Perspectivas latinoamericnas. Consejo Latinoamericano de Ciencias Sociales, CLASCO, UNESCO.

Martínez Guzmán, V. (2005). Filosofia e investigação para a paz. Revista Crítica de Ciências Sociais, Coimbra (Portugal), 71, 43-62.

Martínez Guzmán, V. (2006). Nuevas formas de resolución de conflictos: Transformación, empoderamiento y reconocimiento. Katálysis, 9 (1), 27-37.

PENUD, (2004). Informe sobre desarrollo humano 2004: La libertad cultural en el mundo diverso de hoy. Madrid: PENUD.

Reardon, B. (1985). Sexism and the war system. New York/London: Teachers College, Columbia University.

Sen, A. K. (2000). Desarrollo y libertad. Barcelona: Editorial Planeta.

Stavenhagen, R. (2005). La diversidad cultural en el desarrollo de las Américas. Los pueblos indígenas y los estados nacionales en Hispanoamérica. En Organización de Estados Americanos (OEA). Serie de Estudios Culturales, 9. 
Taylor, C. (1993). El multiculturalismo y "la política del reconocimiento". Comentarios de Amy Gutmann, Steven C. Rockefeller, Michael Walzers y Susan Wolf. México: Fondo de Cultura Económica.

Taylor, C. (1994). Multiculturalism and "the politics of recognition". Princeton, N.J.: Princeton University Press.

UNESCO (1996). Nuestra Diversidad Creativa. Informe de la Comisión Mundial de Cultura y Desarrollo. París: UNESCO.

UNESCO (2001), Declaración sobre la Diversidad Cultural. París: UNESCO.

UNESCO (2005), Convención sobre los Procesos y Promoción de la Diversidad de las Expresiones Culturales. París: UNESCO.

Wallerstein, I. (1996). Después del liberalismo. México: Siglo XXI Editores. 